\title{
Pivoting a large object: whole-body manipulation by a humanoid robot
}

doi:10.1533/abbi.2006.0031

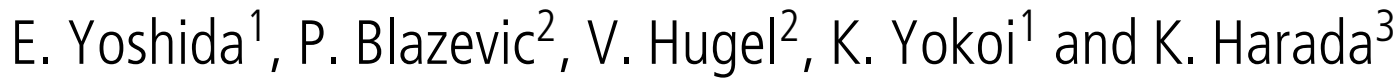 \\ ${ }^{l}$ AIST/ISRI-CNRS/STIC Foint French-Fapanese Robotics Laboratory (FRL), National Institute of Advanced \\ Industrial Science and Technology (AIST), AIST Central 2, Umezono 1-1-1, Tsukuba, Ibaraki 305-8568, Japan \\ ${ }^{2}$ LISV Laboratoire d'Ingénierie des Systèmes de Versailles, 10/12 avenue de l'Europe, 78140 Vélizy, France \\ ${ }^{3}$ Humanoid Research Group, Intelligent Systems Research Institute, National Institute of Advanced Industrial Science \\ and Technology (AIST), AIST Central 2, Umezono 1-1-1, Tsukuba, Ibaraki 305-8568, Japan
}

\begin{abstract}
In this article, we present a method of whole-body manipulation of large objects by a humanoid robot using a pivoting motion. Pivoting manipulation can be an alternative to pushing or lifting for more dexterous and stable operation, which is often used by humans to move large and bulky furniture. This article describes a method and control techniques for a humanoid robot to perform the manipulation of a large object through whole-body motion by applying this pivoting motion. For this purpose, first the object is manipulated by two arms of a humanoid robot using impedance control for grasping, together with body balancing control while the robot stays at the same place. Next, the movement of the humanoid robot itself is performed by stepping motion with the help of resolved momentum control to guarantee the robot stability. The proposed manipulation is validated through dynamic simulation and real hardware experiment of the humanoid robot HRP-2 performing the pivoting task.
\end{abstract}

Key words: Humanoid robot, pivoting, whole-body manipulation.

\section{INTRODUCTION}

Research on humanoid robots has lead to prototypes that can perform complicated tasks such as manipulation, navigation in dynamic environments, or serving tasks.

The manipulation task is one of the promising application areas for humanoid robots since they have a high potential for executing a variety of tasks by fully exploiting their high mobility and adaptability owing to large number of degrees of freedom. Especially, the manipulation of bulky objects through a whole body motion, which has been difficult for other types of robots. This article focuses on the manipulation by a humanoid robot of large objects by introducing pivoting motions (Aiyama et al. 1993). This manipulation has several advantages such as dexterity and stability over other methods like

\footnotetext{
Corresponding Author:

E. Yoshida

AIST/ISRI-CNRS/STIC Joint French-Japanese Robotics Laboratory (JRL)

National Institute of Advanced Industrial Science and Technology (AIST)

AIST Central 2, Umezono 1-1-1

Tsukuba, Ibaraki 305-8568, Japan

Tel: +81298617108; Fax: +81298613443

Email: e.yoshida@aist.go.jp
}

pushing or lifting. Furthermore, the concept of a humanoid manipulating an object in contact with the ground poses interesting research challenges. The dynamic balance of the robot cannot be managed in the same way as in the case of the robot walking simply. The manipulated object brings a new kinematic chain and exerts a reaction force on the robot that must be taken into account. To cope with these problems, position and impedance control framework is introduced first to grasp and manipulate the object, together with a whole-body balancing control. And then, resolved momentum control (RMC) (Kajita et al. 2003 ) is adopted to allow the robot to step forward after manipulation by keeping the hand position with the object.

This article is organized as follows. The next section presents the related work on the subject of manipulation tasks. Section 'Pivoting manipulation algorithm' proposes an algorithm to deal with the manipulation, followed by the description of control techniques included in the algorithm in section 'Control techniques for manipulation and robot motion'. Section 'Simulation' gives simulation results using the dynamic simulator OpenHRP, whose results are verified by hardware experiments using HRP-2 humanoid platform described in section 'Experimental results' before concluding the article. 


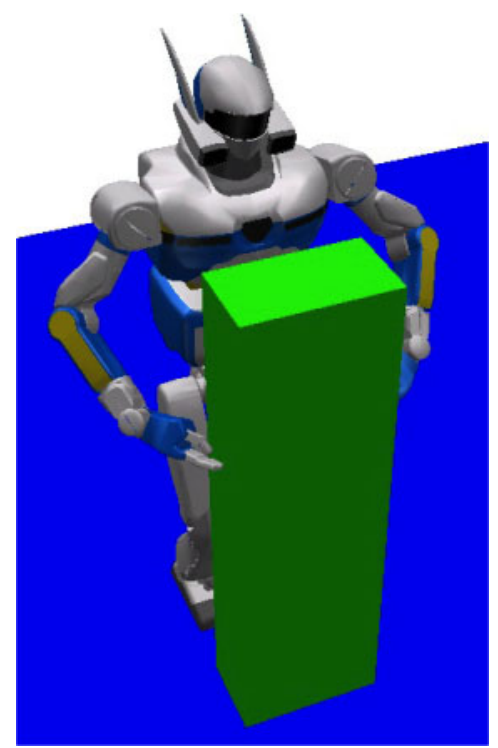

Figure 1 A humanoid robot grasping a box.

\section{RELATED WORK}

For manipulation of large objects that cannot be lifted, we can make use of 'non-prehensile manipulation' methods such as pushing (Mason 1986; Lynch 1992) or tumbling (Bicchi et al. 2004). Aiyama et al. (1993) proposed a graspless manipulation using pivoting motion, whereas Maeda and Arai (2003) reported the analysis and planning.

On the other hand, whole-body manipulation tasks by humanoid robots have been investigated more and more intensely in recent years. The main manipulation methods developed so far are pushing (Hwang et al. 2003; Harada et al. 2004; Takubo et al. 2004) and lifting (Harada et al. 2005).

For a humanoid to manipulate a large object using a whole-body motion, pivoting manipulation (Figure 1), which we humans also often employ, for example, to move an oil drum or a barrel, is considered to have advantages in the following aspects:

- Precise positioning: Since the object is not held in pushing, uncertainty is more significant than pivoting, and also tumbling has limitation in reachability. Although some of these shortcomings can be overcome using sophisticated control methods (Lynch 1992; Bicchi et al. 2004), the robot can move the object to the desired position in a more straightforward manner.

- Adaptability to rough terrains: Pushing is difficult in rough terrains, whereas pivoting can be easily adapted. Lifting and tumbling can be used, but pivoting is more advantageous in terms of flexibility of manipulation and variety in shape and weight of manipulated objects.

- Stability: Pivoting has advantage of lower risk of falling over lifting when manipulating large or heavy objects. Moreover, the manipulated object can help in widening the stability margin in some cases.
Table 1 Comparison of methods of manipulation of a large object by a humanoid ${ }^{\mathrm{a}}$

\begin{tabular}{llll}
\hline & $\begin{array}{l}\text { Precise } \\
\text { positioning }\end{array}$ & Adaptability & Stability \\
\hline Pushing & $\times$ & $\times$ & $\circ$ \\
Lifting & $\circ$ & $\Delta$ & $\times$ \\
Tumbling & $\triangle$ & $\Delta$ & $\circ$ \\
Pivoting & $\circ$ & $\circ$ & $\circ$ \\
\hline
\end{tabular}

$\mathrm{a}_{\circ}$ : suitable; $\Delta$ : can be used; and $\times$ : not suitable.

This comparison is summarized in Table 1 . However, pivoting manipulation has not been fully exploited for humanoid robots due to the difficulty in coordinating manipulating motion with body balancing at whole-body level.

We therefore aim to establish a control method for pivoting manipulation by a whole-body motion of humanoid robot. The aim of this article is two-fold. The first point is the realization of pivoting manipulation that has not been implemented using humanoid platform so far, which provides new potential application fields that require dexterous manipulation by humanoid robots. The second aim is the integration of such control techniques as impedance control for contact force, body balancing during manipulation and RMC for stepping motion, for the purpose of whole-body manipulation. In the following sections, pivoting manipulation by a humanoid robot is presented on the basis of these techniques.

\section{PIVOTING MANIPULATION ALGORITHM}

The manipulation is executed by repeating the following two phases:

- Manipulation of control phase: The manipulation is done in a quasi-static way by repeating rotation of the object about an axis. The contact force to hold the object is controlled using impedance control. The manipulation is performed through whole-body motion coordination to achieve both manipulation and stable body balancing.

- Robot motion phase: The robot moves towards the object with the hands at the same position to continue manipulation. The body motion is planned through RMC.

Figure 2 summarizes the algorithm of the pivoting manipulation task. The detailed manipulation sequence is illustrated in Figure 3, where the dotted and solid lines denote the states before and after the manipulation, respectively.

1. The object is inclined by angle $\alpha$ around an axis $a$, which includes a vertex $v$ on the plane so that the object has a point contact with the plane at $v$ (Figure 3(a)).

2. The object is rotated by angle $\beta$ around the vertical axis $z$ on vertex $v$ to move to the desired direction (Figure 3(b)). As a result, the axis $a$ is rotated to $a^{\prime}$ on the plane. 


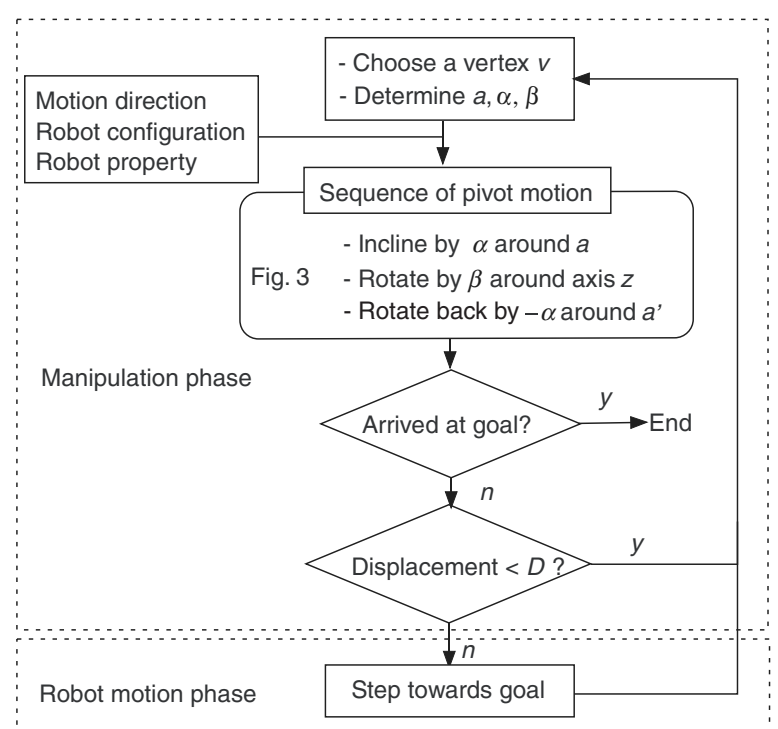

Figure 2 Flow of pivoting manipulation.

3. The object is rotated by $-\alpha$ around the rotated axis $\boldsymbol{a}^{\prime}$ until the front bottom edge of the object touches the ground. The displacement of the object is defined as the distance between the projected points of its centre of mass (CoM) before and after the manipulation (Figure 3(c)).

4. (a) If the displacement of the object exceeds the value $D$, the robot steps toward the desired direction.

5. (b) Otherwise, it continues step 1 or terminates the motion.

The parameters $\alpha, \beta$ and $D$ must be designed so that the manipulated object follows the desired trajectory (Figure 4). The working area and physical properties of the robot body must also be taken into account. The axis $a$ and the vertex around which the object is to be inclined are selected in order not to lose the stability of the robot and the object. In our case, the vertex closer to the robot is selected since smaller torque is required for the movement, and the direction of axis $a$ is chosen such that the robot does not make a large forward motion.

The axis $z$ is set to the vertical axis since no work is required for quasi-static motion around this axis. The angle $\beta$ depends on the constraint of robot motion capability as well as the desired trajectory of the robot. The hand

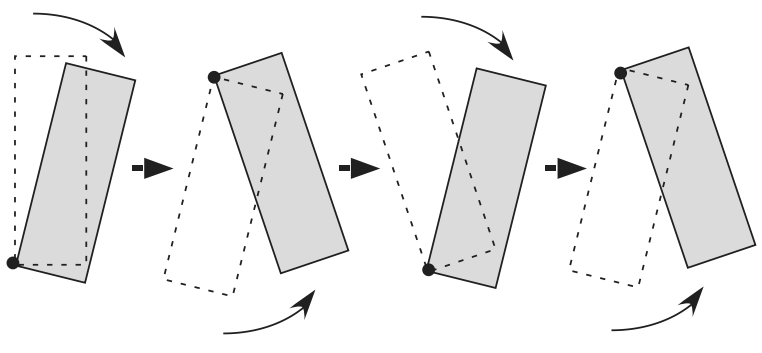

Figure 4 Object transporting by repeated pivoting.

trajectories can be calculated geometrically from the pivoting motion and contact points of the hand on the object.

To execute more complicated trajectory than that given in Figure 4, we will further need a strategy to determine these parameters by fully realizing the combination of stepping motion.

The next section details the manipulation control and robot motion for the pivoting task.

\section{CONTROL TECHNIQUES FOR MANIPULATION AND ROBOT MOTION}

In this section, we describe how a humanoid robot controls the manipulation task. In the manipulation control phase, the robot is supposed to firmly grasp the object by two hands (Figure 1) without slipping. First, we introduce impedance control to manipulate the contact force to hold the object. Then, a method of body balancing is adopted to maintain the CoM in the supporting phase during manipulation. We assume that the manipulation is done for a rectangular box-shaped object in a quasi-static manner by repeating the rotations on a plane as described in Figure 3.

\section{Grasping}

Since we assume quasi-static motion, a position control for robot hands to achieve the trajectory for the desired motion is adopted. For position-controlled robot such as HRP-2 used for simulations and experiments, the output of the following impedance control is added to the position command of manipulation to regulate the contact force (Harada et al. 2004).

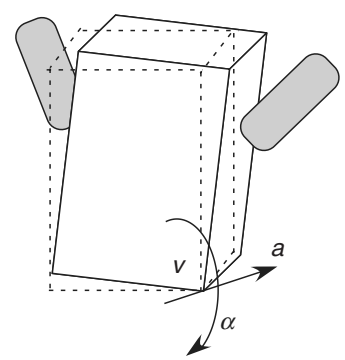

(a) Inclining

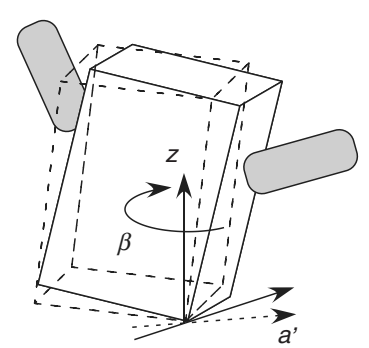

(b) Rotating around $z$ axis

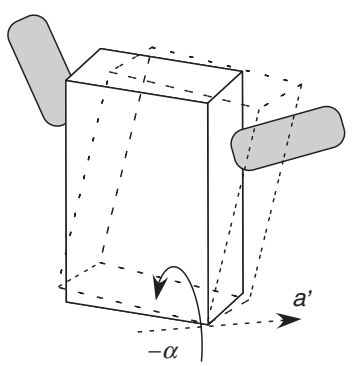

(c) To initial contact

Figure 3 Sequence of pivot motion of a box object: (a) inclining; (b) rotating around $z$ axis; and (c) to initial contact. 


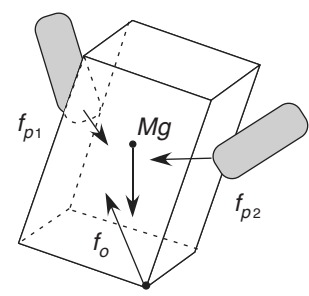

Figure 5 Forces for pivot motion.

The robot hands are placed on the two sides of the object so that they can exert forces in the opposite direction. Let $x_{H}$ be the hand position that is controlled in the direction perpendicular to the object face, and let $f_{x}$ be the force exerted to the hand. The impedance control law to lead the force $f_{x}$ to the desired force $f_{x d}$ is given as

$$
m \ddot{x}_{H}+c \dot{x}_{H}=f_{x d}-f_{x},
$$

where $m$ and $c$ are the mass and damper parameters. For grasping, this law governs manipulation of one of the hands, and the other is controlled in position to avoid unnecessary oscillation. The control law is discretised in the simulation and experiment implementation.

\section{Pivoting manipulation}

In our case, the robot holds the object rigidly by two hands, as illustrated in Figure 5. Let $p_{p i}(i=1,2)$ be the vector of position of each hand, $p_{g}$ be the position of the centre of gravity and $f_{p i}$ be the force applied to each grasping point. The object is in static equilibrium when the following conditions are satisfied:

$$
\begin{array}{r}
f_{o}+\sum_{i=1}^{2} f_{p i}+M g=\boldsymbol{o}, \\
\sum_{i=1}^{2} p_{p i} \times f_{p i}+p_{g} \times M g=\boldsymbol{o},
\end{array}
$$

where $M g$ and $f_{o}$ are the gravity vector and resistance force at the contact point, respectively. In this study, we assume that the manipulation is executed quasi-statically and that no slipping occurs at the contact points. We also assume that the geometry and location of the object are known. The object is manipulated by moving each hand under the above condition. The contact forces are controlled by impedance control (1) by giving the desired contact force $f_{x d}$.

\section{Whole-body balancing}

During the manipulation task, the robot should not only apply the necessary force to the hand for manipulation but also keep the balance of the whole body.

We adopt the method developed by Harada et al. (2005). The key idea is to control the waist position in order so that the 'static balancing point' is on the centre of the foot-supporting polygon. The static balancing point $p_{s}=$ $\left[x_{s}, y_{s}, 0\right]^{\mathrm{T}}$ is the point on the floor to which all the

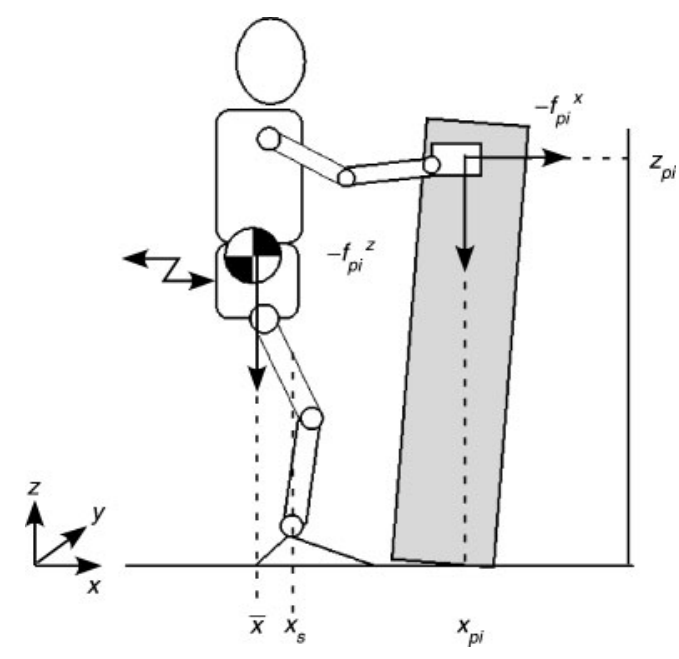

Figure 6 Body balancing by moving waist position.

resistance force from both hands and gravity are applied, which is equivalent to zero moment point (ZMP) when no dynamic motion is generated. This is described as:

$$
\begin{aligned}
& x_{s}-\bar{x}=-\sum_{i=1}^{2} \frac{z_{p i} f_{p i}^{x}+\left(x_{p i}-x_{s}\right) f_{p i}^{z}}{M_{R} g} \\
& y_{s}-\bar{y}=-\sum_{i=1}^{2} \frac{z_{p i} f_{p i}^{y}+\left(y_{p i}-y_{s}\right) f_{p i}^{z}}{M_{R} g}
\end{aligned}
$$

where $f_{p i}=\left[f_{p i}^{x}, f_{p i}^{y} f_{p i}^{z}\right]^{\mathrm{T}}(i=1,2), p_{p i}=\left[x_{p i}, y_{p i}, z_{p i}\right]^{\mathrm{T}}$ and $g=[0,0,-g]^{\mathrm{T}} \cdot \bar{p}=[\bar{x}, \bar{y}, \bar{z}]^{\mathrm{T}}$ denotes the position of the CoM without external force and $M_{R}$ is the total mass of the robot. Note that the sign in right-hand side of (4) and (5) is negative since the reaction force at each hand is $-f_{p i}$.

The balancing control is performed by moving the position of the waist so that the position of the static balancing point $p_{s}$ is at a desired position inside the convex hull of supporting area(s) (Figure 6). The movement of the waist position can be approximated by the CoM position $\bar{p}$ from the desired position $p_{d}$ of $p_{s}$ (Harada et al. 2005).

In this way, the force of pivoting manipulation is generated as a resulting compensation of disturbance due to the contact with the object.

\section{Robot stepping motion}

After a sequence of manipulation, the object will be displaced to a farther position from the robot. Now the robot itself needs to move in the desired direction of object transportation. It is, therefore, preferable that the robot keeps the hands on the object so to be easily manipulated.

Here, we introduce RMC, which is a control framework, where a humanoid robot is controlled such that the resultant total linear/angular momenta reach specified values (Kajita et al. 2003). It is well known that, for stable walking in a humanoid robot, the ZMP must be within the convex hull of the supporting area(s). By using RMC, we can control the CoM of the robot so that it is always within 


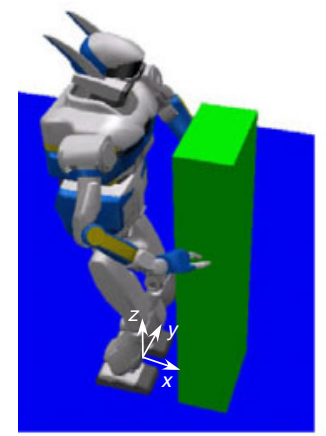

(a)

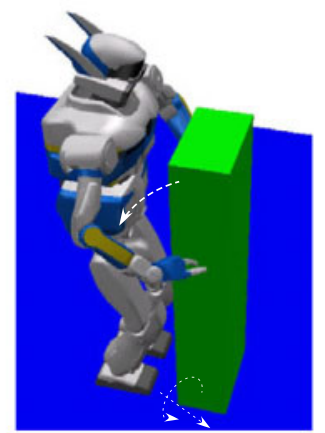

(b)

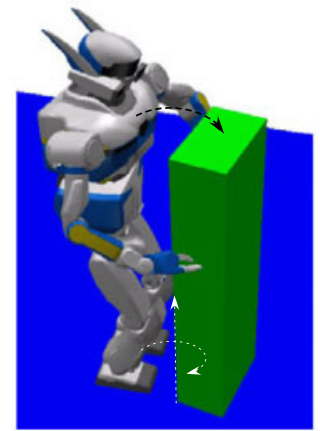

(c)

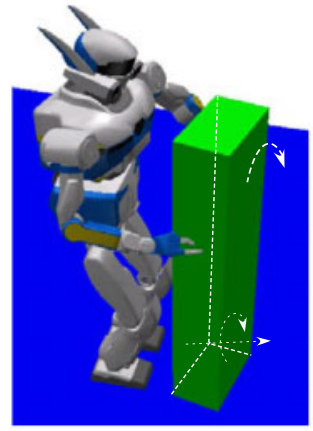

(d)

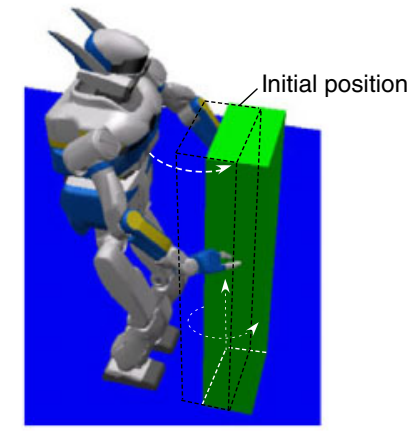

(e)

Figure 7 Simulation results of pivoting motion: (a) initial state; (b) Step 1: inclining rightward; (c) Step 3: rotating clockwise; (d) Step 4: inclining leftward; and (e) Step 5: rotating counterclockwise.

Table 2 Parameters of pivot motion

\begin{tabular}{lllllll}
\hline Step & $\begin{array}{l}\text { Type (in } \\
\text { Figure 3) }\end{array}$ & Axis $^{\mathrm{a}}$ & Angle $\left(^{\circ}\right)$ & Time (s) & $\begin{array}{l}\text { Contact } \\
\text { vertex }^{\mathrm{b}}\end{array}$ & Description $^{\mathrm{b}}$ \\
\hline 1 & $(\mathrm{a})$ & $a_{1}=(3,-1,0)$ & $\alpha=5.0$ & 1.0 & NR & Inclining rightward \\
2 & $(\mathrm{~b})$ & $z=(0,0,1)$ & $\beta=-15.0$ & 3.0 & NR & Rotating CW \\
3 & $(\mathrm{c})$ & $a_{1}^{\prime}=(3,-1,0)^{\mathrm{c}}$ & $\alpha=-5.0$ & 1.0 & NR & Inclining back \\
4 & $(\mathrm{a})$ & $a_{2}=(3,1,0)$ & $\alpha=-5.0$ & 1.0 & NL & Inclining leftward \\
5 & $(\mathrm{~b})$ & $z=(0,0,1)$ & $\beta=15.0$ & 3.0 & NL & Rotating CCW \\
6 & (c) & $a_{2}^{\prime}=(3,1,0)^{\mathrm{c}}$ & $\alpha=5.0$ & 1.0 & NL & Inclining back \\
\hline
\end{tabular}

${ }^{a}$ See Figure 7(a) for the reference frame. The angle between $x$ and $a_{1}$ and $a_{2}$ is $18.4^{\circ}$ and $-18.4^{\circ}$, respectively.

${ }^{\mathrm{b} N R}$ : near-right; NL: near-left; CW: clockwise; and CCW: counterclockwise.

${ }^{\mathrm{c}} \mathrm{Axis} \boldsymbol{a}^{\prime}$ is rotated vector $a$ around $z$ by $\beta$.

the convex hull of the supporting area(s) to maintain the stability of the robot. Since the linear momentum $P$ depends on the time derivative of CoM position $r$ through the total mass $M_{R}$ as $P=M_{R} \dot{p}_{g}$, the position of CoM can be controlled by manipulating the linear momentum as $P=k M_{R}\left(\tilde{p}_{g}-p_{g}\right)$, where the tilde denotes the reference value, and $k$ is the gain of the control scheme. Using this equation, we are can calculate the desired linear momentum $P$ to control CoM of the robot.

The hand position can be controlled on the basis of an extended RMC method developed by Neo et al. (2003) by keeping the position of CoM inside the supporting area.

In this way, the robot can step towards the object with both its hands at contact positions, whereas the CoM is controlled at all times inside the convex hull of supporting area(s). Moreover, keeping the hand position on the object may help to maintain the equilibrium of the robot body.

\section{SIMULATION}

We have conducted a simulation of the proposed pivoting motion using the humanoid robot simulator OpenHRP (Kanehiro et al. 2001) where the results are verified by using humanoid robot HRP-2 (Kaneko et al. 2004). HRP2 has 30 degrees of freedom, with $1.54-\mathrm{m}$ height and $58 \mathrm{~kg}$ weight. Wrists and ankles are equipped with force sensors. Since the input of force sensor is noisy, we used an average value of the last 20 samplings measured every $5 \mathrm{~ms}$. The width, depth and height of the object are $0.35 \mathrm{~m}, 0.2 \mathrm{~m}$ and $1.2 \mathrm{~m}$, respectively. The mass is $3 \mathrm{~kg}$. 


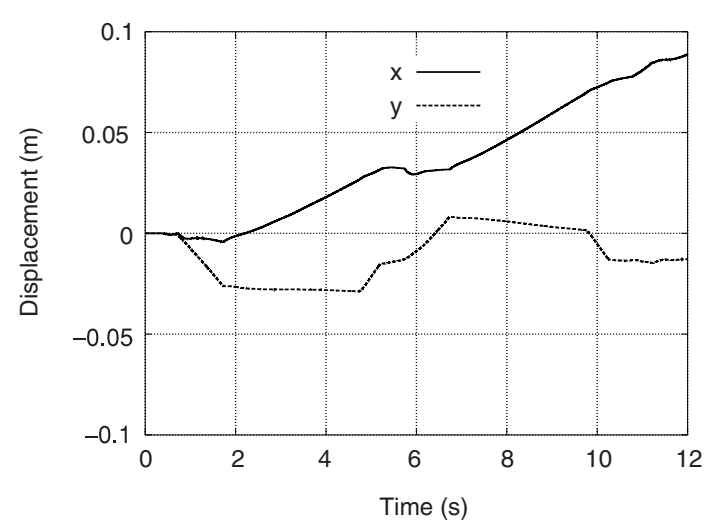

Figure 8 Displacement of the manipulated object.

The parameters of the pivoting sequence are shown in Table 2. The directions of axes are given in Figure 7(a). Here the parameter $D$ is set at $50 \mathrm{~mm}$ to prevent the arms from going into singular configuration and losing stability by too much extension of the arm. For the impedance control, we assume $m=10 \mathrm{~kg}, c=300 \mathrm{~N} \cdot \mathrm{m}^{-1} \cdot \mathrm{s}$ and $f_{x d}=$ $25 \mathrm{~N}$, respectively. The contact force $f_{x d}$ for the robot to hold firmly to prevent the object from slipping because of gravity is determined. The $z$ position of contact point is set at $0.62 \mathrm{~m}$ and at the midpoint of the depth of the object in the $x$ direction.

In the simulation, first the robot grasps the object firmly and stays at the original position until the contact force by impedance control stabilizes. The hand trajectories during the manipulation are computed geometrically by using the contact points of both hands and the motions described in Table 2. Position control is performed to follow the calculated hand trajectories and the impedance control law (1) is applied to one of the hands to maintain grasping during the manipulation.

Figure 7 shows the snapshots of the simulation. As can be seen, the humanoid robot successfully displaces the object by pivoting.

We measure the displacement of the manipulated object, the contact forces and CoM of the robot, to evaluate respectively the performance of the manipulation itself, impedance control and the balance control.

Figure 8 shows the displacement of the CoM of the manipulated object projected on the plane. Since the object is first inclined around the axis $a$ before rotating around the axis $z$, the displacement $x$ reduces first and then increases. This occurs twice, and the object is displaced by $0.073 \mathrm{~m}$ in the $x$ direction.

Figure 9 shows the simulations of the contact forces $f p_{1}$ and $f p_{2}$ for both hands. As shown in the figure, the contact occurs at the very beginning when both hands are approaching the object. Then, both the contact forces stabilize around the desired force $f_{x d}=25 \mathrm{~N}$ at the beginning of the manipulation. The contact force decreases during the manipulation. This is because the arms are in a stretched configuration where it is difficult to generate the

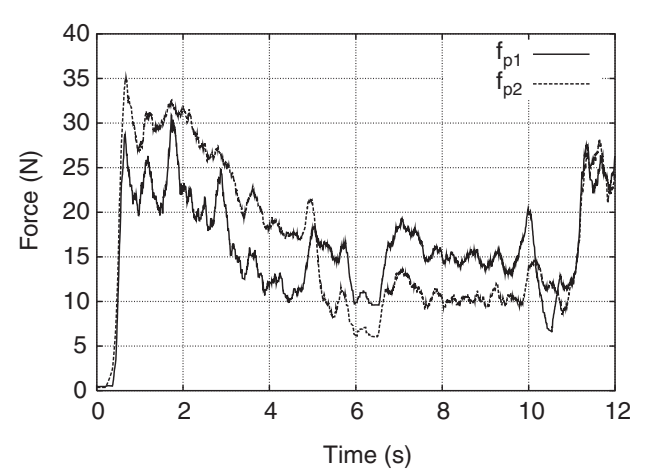

Figure 9 Contact forces of each hand.

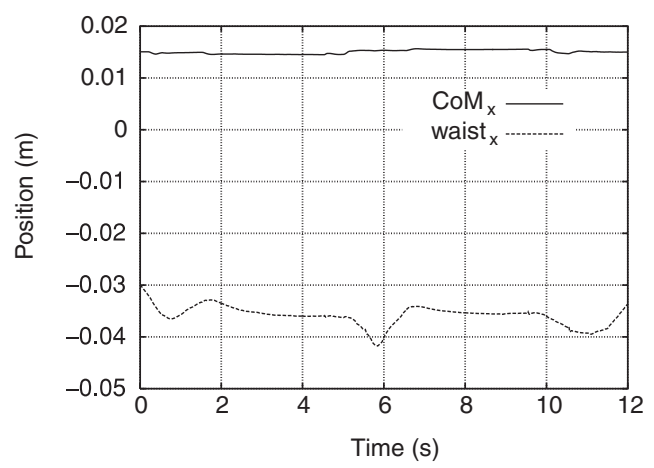

Figure 10 Centre of mass and waist positions.

force in the desired direction during manipulation, especially for the position-controlled arm. However, the forces regain the desired value after the manipulation. This simulation result verifies that the usage of impedance control for contact force is sufficiently effective.

The $x$ coordinates of static balancing point $p_{s}$ and CoM position $\bar{p}$ without external force, $x_{s}$ and $\bar{x}$, are plotted in Figure 10 . Here $\bar{p}$ represents an approximated movement of the waist position. Without balancing control, $x_{s}$ would keep increasing as the robot extends the arms and force is applied to the hand during the manipulation until the robot falls down. In the graph, the static balancing point is regulated by moving the waist position so that it coincides with the centre of the feet (shift along $x$ axis of $0.015 \mathrm{~m}$ ) according to the balancing control.

The robot motion phase is simulated on the basis of the proposed method. Figure 11 shows the sequence of the stepping forward motion. After the manipulation, the robot steps forward by $50 \mathrm{~mm}$ using alternative foot movements, by keeping the hand on the object using RMC to maintain the whole-body balance. As shown in the figure, robot first moves its CoM on the right foot, and then moves the left foot forward. The same sequence is repeated for the right foot. The simulation shows that the robot can effectively move towards the desired direction of manipulation.

\section{EXPERIMENTAL RESULTS}

We have conducted an experiment of the manipulation control in the same condition as in simulation using HRP-2 


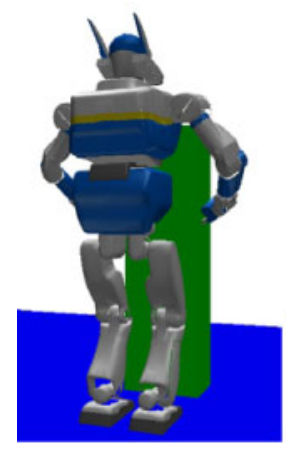

Initial position

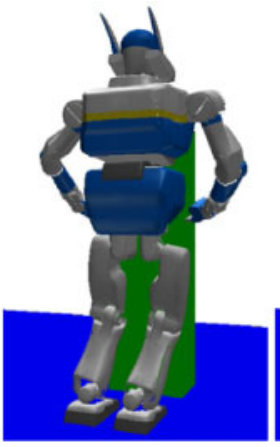

Moving CoM rightward

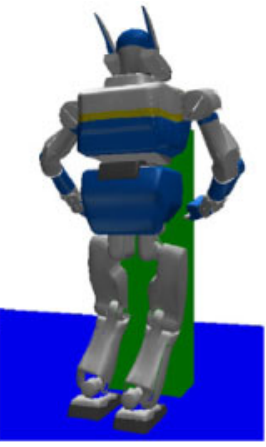

Stepping left leg

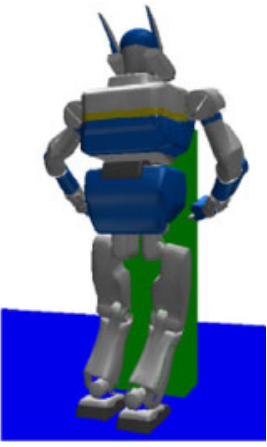

Left leg landed

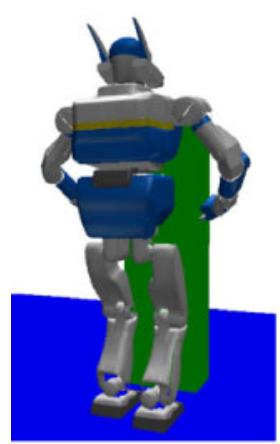

Moving CoM center

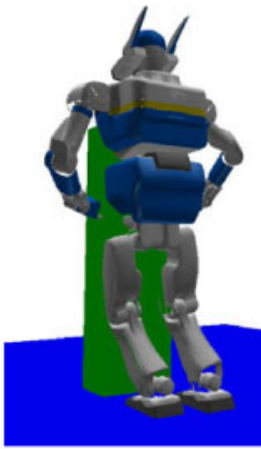

Moving CoM leftward

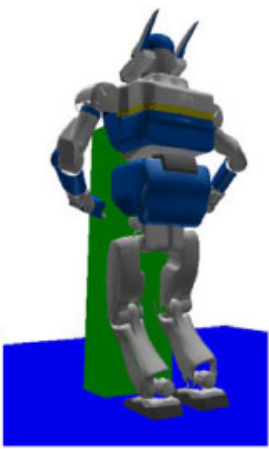

Stepping right leg

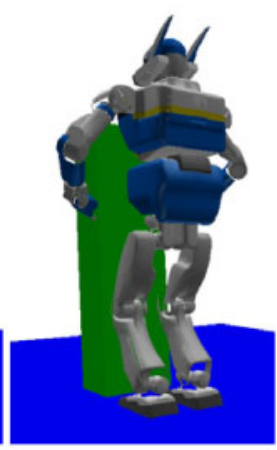

Stepping finished

Figure 11 Stepping forward keeping the hands on the object.

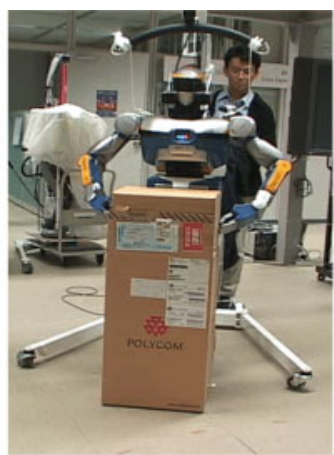

(a)

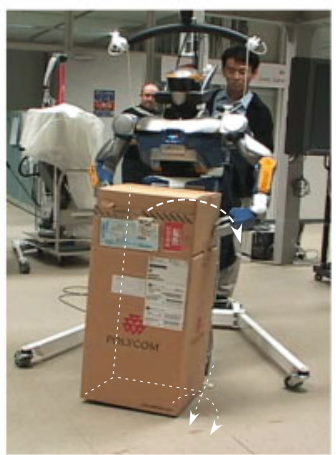

(d)

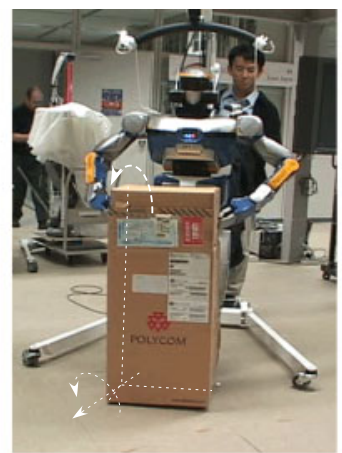

(b)

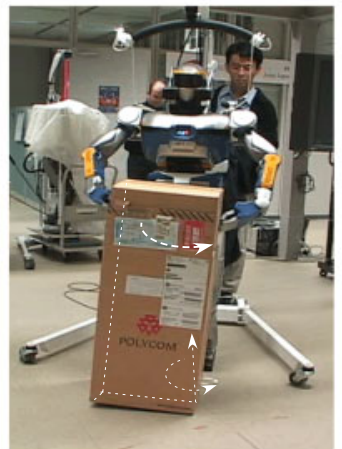

(e)

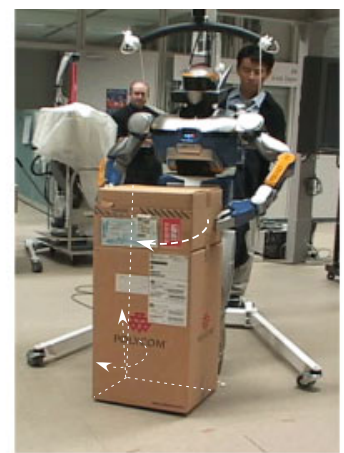

(c)

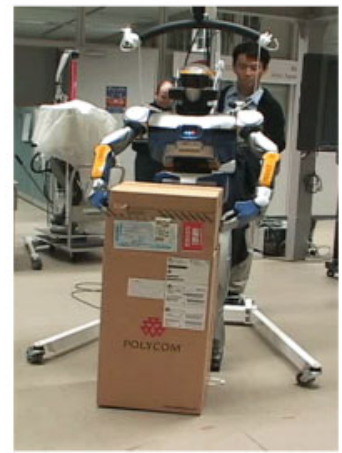

(f)

Figure 12 Experiment of pivoting motion: (a) initial state; (b) Step 1: inclining rightward; (c) Step 3: rotating clockwise; (d) Step 4: inclining leftward; (e) Step 5: rotating counterclockwise; and (f) final state. 


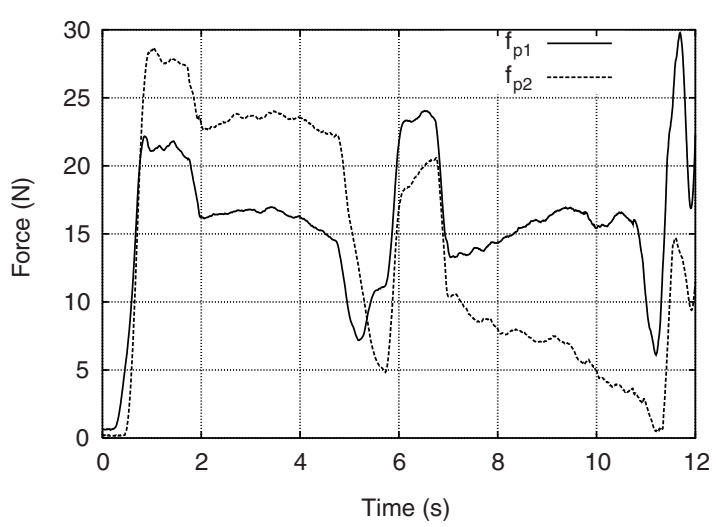

Figure 13 Experimental result of contact forces of each hand.

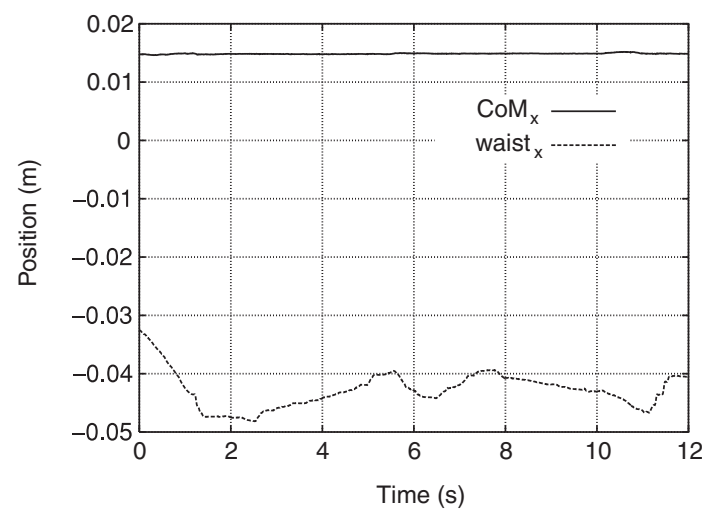

Figure 14 Experimental result of centre of mass and waist positions.

humanoid hardware platform. Owing to the architecture of OpenHRP, the developed simulation software has binary compatibility with the robot hardware.

Figure 12 shows snapshots of the experiments using almost the same size and weight of the box as in the simulation. As can be seen, the pivoting manipulation has been executed appropriately, and the displacement in the $x$ direction was around $0.06 \mathrm{~m}$ as expected from simulation.

Figure 13 shows the measurement of contact forces from wrist force sensors. Although the measured forces show similar characteristics with the simulation, one of the forces drops drastically at the end of manipulation. The manipulation experiment was successful; however, the arm configuration and grasping position need to be investigated for more reliable manipulation. Similar experimental results as the values given in the graph in Figure 10 show the effectiveness of balance control to keep the static balancing point in stable position as shown in Figure 14.

\section{CONCLUSION}

In this article, we have presented a pivoting manipulation as an application of a humanoid robot platform. By realizing this dexterous manipulation that enables precise displacement of heavy or bulky objects, the application area of humanoid robots can be significantly extended.
We have proposed a sequence of pivoting motion composed of two phases, manipulation control and robot motion. In the former phase, impedance control and balancing control were introduced to control the required contact force for grasping and to maintain stability during manipulation, respectively. Framework of RMC was adopted for stepping motion in the latter phase.

Thus, we showed a sequence of pivoting motion to manipulate the objects towards the desired direction. We have shown that the proposed pivoting manipulation can be effectively realized by computer simulation and experiments using a humanoid robot platform HRP-2.

In pursuit of wide application in future developments, improvement in the method to adapt to various shapes of the object of transportation would be undertaken. Another future extension is the manipulation strategy for more general trajectories with experimentation of both manipulation and stepping phases. Integration of identification of objects or environments in contact ( $\mathrm{Yu}$ et al. 1999; Debus et al. 2000) with the robot is also an important issue for the improvement of the robot's dexterity.

\section{ACKNOWLEDGMENTS}

The authors express their sincere gratitude to Dr. Shuuji Kajita of Humanoid Research Group, Intelligent Systems Research Institute, AIST, for his helpful advice about the control issues. The authors also acknowledge Prof. Yasumichi Aiyama of Tsukuba University for his references to state of the art in the research field of manipulation.

\section{REFERENCES}

Aiyama Y, Inaba M, Inoue H. 1993. Pivoting: a new method of graspless manipulation of object by robot fingers. In Proceedings of the IEEE/RSJ International Conference on Intelligent Robots and Systems, 26-30 July 1993, Yokohama, Japan, p. 136-43.

Bicchi A, Chitour Y, Marigo A. 2004. Reachability and steering of rolling polyhedra: a case study in discrete nonholonomy. IEEE Trans Autom Control, 49(5), 710-26.

Debus T, Dupont P, Howe R. 2000. Automatic identification of local geometric properties during teleoperation. In Proceedings of IEEE International Conference on Robotics and Automation, 24-28 April 2000, San Fransisco, USA, p. 3428-34.

Harada H, Kajita S, Kanehiro F, et al. 2004. Real-time planning of humanoid robot's gait for force controlled manipulation. In Proceedings of IEEE International Conference on Robotics and Automation, 26 April-1 May 2004, New Orleans, USA, p. 616-22.

Harada H, Kajita S, Saito H, et al. 2005. A humanoid robot carrying a heavy object. In Proceedings of IEEE International Conference on Robotics and Automation, 18-22 April 2005, Barcelona, Spain, p. 1724-9.

Hwang Y, Konno A, Uchiyama M. 2003. Whole body cooperative tasks and static stability evaluations for a humanoid robot. In Proceedings of the IEEE/RSJ International Conference on 
Intelligent Robots and Systems, 27 October-1 November 2003, Las Vegas, USA, p. 1901-6.

Kajita S, Kanehiro F, Kaneko K, et al. 2003. Resolved momentum control: humanoid motion planning based on the linear and angular momentum. In Proceedings of the IEEE/RSJ International Conference on Intelligent Robots and Systems, 27 October-1 November 2003, Las Vegas, USA, p. 1644-50.

Kanehiro F, Miyata N, Kajita S, et al. 2001. Virtual humanoid robot platform to develop. In Proceedings of the IEEE/RSJ International Conference on Intelligent Robots and Systems, 29 October-3 November 2001, Hawaii, USA, p. 1093-9.

Kaneko K, Kanehiro F, Kajita S, et al. 2004. The humanoid robot HRP-2. In Proceedings of the IEEE/RSJ International Conference on Robotics and Automation, 26 April-1 May 2004, New Orleans, USA, p. 1083-90.

Lynch K. 1992. The mechanics of fine manipulation by pushing. In Proceedings of the IEEE International Conference on Robotics and Automation, 1992, p. 2269-76.

Maeda Y, Arai T. 2003. Automatic determination of finger control modes for graspless manipulation. In Proceedings of the IEEE/RSJ International Conference on Intelligent Robots and Systems, 27 October-1 November 2003, Las Vegas, USA, p. $2660-5$.
Mason M. 1986. Mechanics and planning of manipulator pushing operation. Int $\mathcal{7}$ Rob Res, 5(3), 53-71.

Neo ES, Yokoi K, Kajita S, et al. 2003. Whole body teleoperation of a humanoid robot integrating operator's intention and robot's autonomy - an experimental verification. In Proceedings of the IEEE/RSJ International Conference on Intelligent Robots and Systems, 27 October-1 November 2003, Las Vegas, USA, p. 1651-6.

Takubo T, Inoue K, Sakata K, et al. 2004. Mobile manipulation of humanoid robots control method for com position with external force. In Proceedings of the IEEE/RSJ International Conference on Intelligent Robots and Systems, 28 September-2 October 2004, Sendai, Japan, p. 1180-5.

Yoshida H, Inoue K, Arai T, et al. 2002. Mobile manipulation of humanoid robots-optimal posture for generating large force based on statics. In Proceedings of the IEEE International Conference on Robotics and Automation, 11-15 May 2002, Washington, DC, p. 2271-6.

Yu Y, Fukuda K, Tsujio S. 1999. Estimation of mass and center of mass of graspless and shape-unknown object. In Proceedings of the IEEE International Conference on Robotics and Automation, 10-15 May 1999, Detroit, USA, p. 2893-8. 

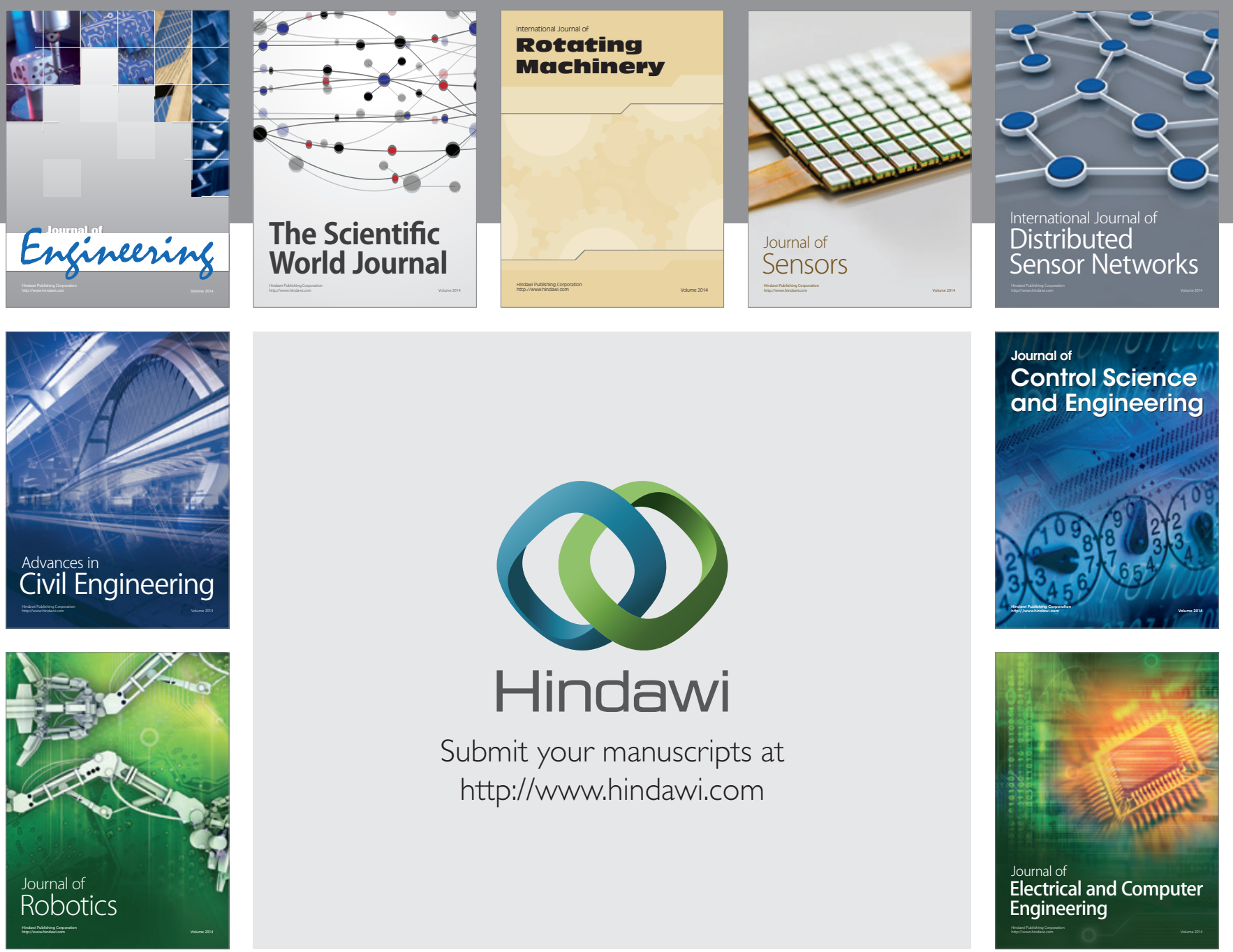

Submit your manuscripts at

http://www.hindawi.com
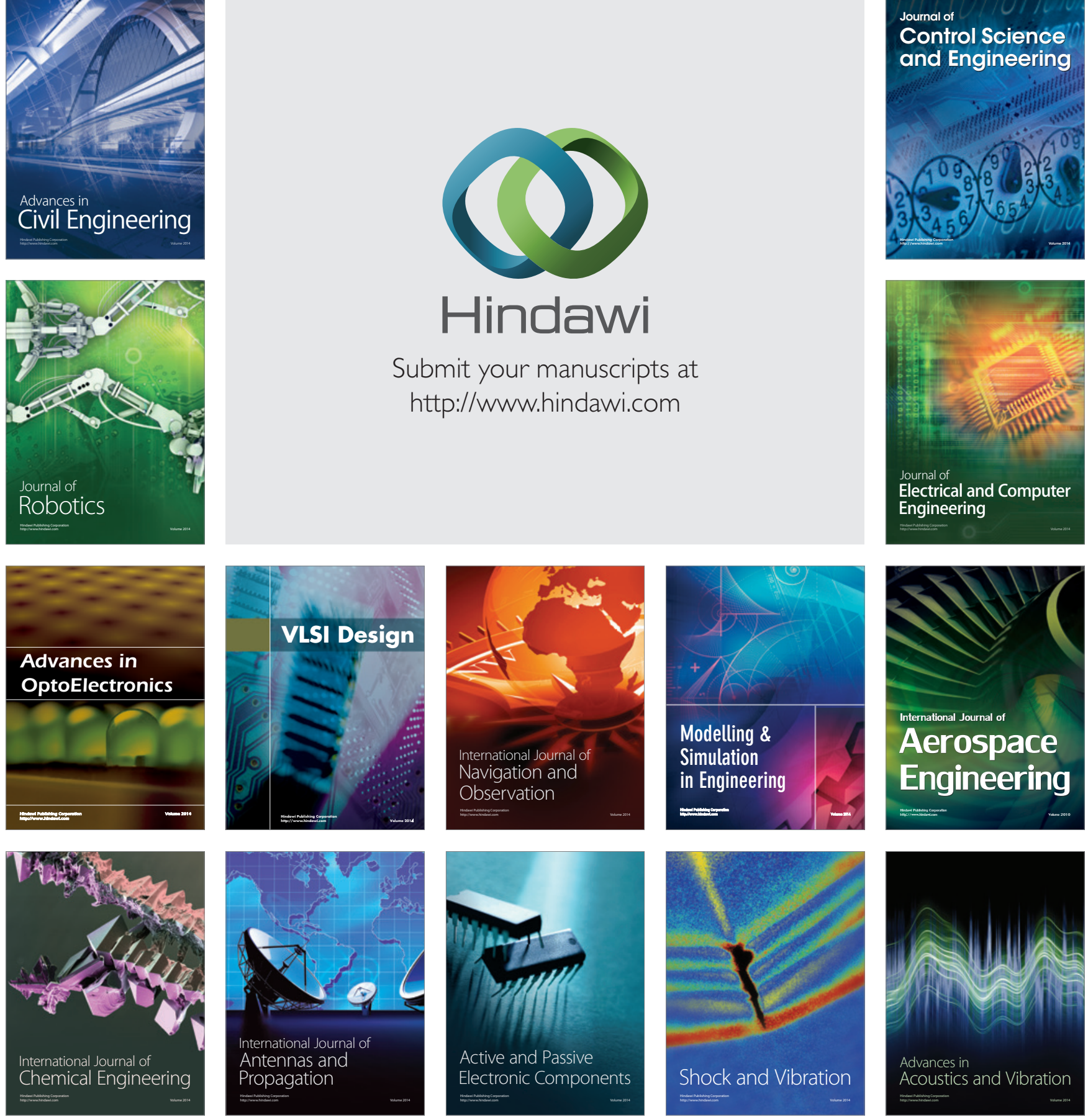